\title{
A Functional $S$ Locus Anther Gene is Not Required for the Self-Incompatibility Response in Brassica oleracea
}

\author{
Martine Pastuglia ${ }^{\mathrm{a}}$, Véronique Ruffio-Châble ${ }^{\mathrm{b}}$, Valérie Delorme ${ }^{\mathrm{a}, 1}$, Thierry \\ Gaude $^{\mathrm{a}}$, Christian Dumas ${ }^{\mathrm{a}}$ and J. Mark Cock ${ }^{\mathrm{a}, 2}$
}

a Reproduction et Développement des Plantes, UMR 9938 CNRS-INRA-ENSL, Ecole Normale Supérieure de Lyon, 46 allée d'Italie, 69364 Lyon Cedex 07, France

${ }^{\mathrm{b}}$ Amélioration des Plantes, Institut National de la Recherche Agronomique - Domaine de la Motte, BP29, 35650 Le Rheu Cedex, France

${ }^{1}$ Current address: Department of Plant Sciences, University of Oxford, South Parks Road, Oxford OX1 3RB, United Kingdom.

RUNNING TITLE: Self-incompatible SLA mutants 
${ }^{2}$ To whom correspondence should be addressed. E-mail: Mark.Cock@ens-lyon.fr; fax 33-472-72-86-00. 


\section{ABSTRACT}

The self-incompatibility (SI) response in Brassica involves recognition of self-pollen by the papillar cells of the stigma and is mediated by the products of genes localized at the $S$ (selfincompatibility) locus. Two $S$ locus genes, $S R K$ and $S L G$, are thought to encode components of a receptor complex present in the female partner. The putative gene product of $S L A$, a third $S$ locus-linked gene that is expressed specifically in anthers, is a candidate for the male component of the SI recognition system. The identification of a mutant SLA allele, interrupted by a large insert resembling a retrotransposon, in self-compatible Brassica napus initially suggested that SLA played an essential role in the SI response. In this study, we have characterized an SLA allele from a self-compatible B. oleracea var acephala line and show that it too is interrupted by a large insert. However, analysis of seven B. oleracea var botrytis lines exhibiting both self-compatible and self-incompatible phenotypes showed that these lines carry an $S$ allele very similar or identical to that of the B. oleracea var acephala line and that the SLA gene is interrupted by an insert in all seven lines. The insertion of the putative retrotransposon was shown to interfere with gene expression, with no SLA transcripts being detected by RNA gel blot analysis in a self-incompatible $B$. oleracea var botrytis line carrying an interrupted $S L A$ gene. These data indicate that a functional SLA gene is not required for the self-incompatibility response in Brassica.

\section{INTRODUCTION}

During compatible pollination in Brassica, pollen grains adhere to the papillar cells of the stigma, hydrate, and then germinate to emit a pollen tube. The pollen tube grows down through the transmitting tissue carrying the male gametes, which fertilize an ovum in an 
ovary at the base of the pistil. In self-incompatible plants, self-pollen grains are recognized by the papillar cells, and the pollination process is rapidly blocked, usually before the hydration or germination stage (reviewed in Nasrallah et al., 1994b; McCubbin and Kao, 1996).

Self-incompatibility (SI) in Brassica is controlled by a single, highly polymorphic locus, the $S$ locus, of which there are more than 50 alleles (Ockendon, 1974, 1982). Rejection of self-pollen occurs when the same $S$ allele is expressed in both pollen and stigma. The effectiveness of the SI response varies depending on which $S$ alleles are present and the dominance/recessivity relationships that exist between different $S$ alleles. Based on these phenomena, $S$ alleles have been broadly classified into two groups depending on whether they tend to be dominant or codominant and confer a strong SI response (class I) or whether they tend to be recessive and confer a weak SI response (class II).

The $S$ locus has a complex molecular structure. A number of genes have been shown to be genetically linked to the $S$ locus: $S L G$ (for $S$ locus glycoprotein; Nasrallah et al., 1985, 1987), SRK (for $S$ locus receptor kinase; Stein et al., 1991), SLA (for $S$ locus anther; Boyes and Nasrallah, 1995), 298 and 299 (Boyes et al., 1997), and $S L L_{1}$ and $S L L_{2}$ (for $S$ locus-linked genes; Yu et al., 1996). Moreover, pulse field gel electrophoresis analysis suggests that the polymorphic nature of the $S$ locus may extend to a region of up to $570 \mathrm{~kb}$ surrounding the $S$ locus genes (Boyes and Nasrallah, 1993). As a result of this complex structure and the fact that several of the $S$ locus genes have been implicated in the SI response, the term "S haplotype" is used rather than "S allele" to describe different allelic forms of the $S$ locus (Boyes and Nasrallah, 1993).

Two $S$ locus genes, $S L G$ and the $S R K$, are thought to be involved in perception of selfpollen by the stigma. Both genes are highly polymorphic, and both are expressed specifically at the surface of mature stigmas. Low levels of mRNA for these two genes are also present in anthers (Sato et al., 1991; Stein et al., 1991), but the protein products of the genes have not 
been detected in this tissue (Delorme et al., 1995b; Stein et al., 1996). Sequence data are available for several different alleles of both $S L G$ and $S R K$. These genes exhibit the high level of polymorphism expected for genes involved in the SI response and, moreover, the different alleles of $S L G$ and $S R K$ can be divided into two classes based on sequence similarities, which correspond closely with the two classes defined on the basis of phenotype. Further evidence that $S R K$ is involved in the SI response has come from the identification of self-compatible Brassica plants that also possess mutated SRK genes (Goring et al., 1993; Nasrallah et al., 1994a).

SLG encodes an abundant, secreted glycoprotein (Nasrallah et al., 1985, 1987), whereas $S R K$ encodes a membrane-spanning protein kinase with a predicted extracellular domain that shares extensive sequence identity (around 90\%) with SLG (Stein et al., 1991; Delorme et al., 1995b). SRK is a member of the large receptor-like kinase (RLK) gene family that has been identified in plants (Becraft et al., 1996; Braun and Walker, 1996; Hervé et al., 1996; Schulze-Muth et al., 1996; Swarup et al., 1996; Wang et al., 1996). The different gene products encoded by members of the RLK superfamily are thought to function as receptors based on their similarity to animal receptor kinases, but this has yet to be demonstrated experimentally. The $S R K$ gene product is perhaps the best characterized member of this family. Monoclonal antibodies raised against a peptide based on the SRK sequence detected a $120-\mathrm{kD}$ protein in stigma extracts, and this protein has been shown to be glycosylated and anchored in the plasma membrane (Delorme et al., 1995b; Stein et al., 1996). The kinase domain of SRK has been expressed in Escherichia coli and has been shown to have protein serine/threonine kinase activity (Goring and Rothstein, 1992). A soluble, truncated form of SRK (eSRK for extracellular form of SRK) has also been detected in stigma extracts (Giranton et al., 1995). This protein, which is produced as a result of alternative splicing of 
SRK transcripts, lacks the membrane-spanning and kinase domains of SRK and therefore closely resembles SLG.

Current models describing the pollen-pistil recognition step of the SI response propose that SRK, present in the cell membrane of papillar cells on the stigma surface, binds to a pollen-borne ligand and initiates a protein phosphorylation cascade that leads to the SI response. SLG and eSRK are thought to modulate signal transduction via SRK. Identification of a ligand bound by SRK would greatly facilitate testing of these models. To date, none of the proteins encoded by members of the RLK superfamily have been shown to bind a ligand (hence the term receptor-like kinase) although, in the case of SRK, two candidate ligands have been proposed in the literature.

The first candidate is a small protein ( $\mathrm{PCP} 7$ for $7-\mathrm{kD}$ pollen coat protein) present on the surface of Brassica pollen (in the pollen coat). It has been shown to interact with SLG in vitro (Doughty et al., 1993). It is thought that PCP7 or a related protein might therefore bind to SRK, either directly as a result of SRK's similarity with SLG, or indirectly as a complex incorporating SLG. However, no PCP has yet been shown to bind specifically to the gene products of a particular $S$ haplotype and, although a cDNA clone has been isolated for one member of the $P C P$ gene family, no members of this gene family have yet been shown to be linked to the $S$ locus (Stanchev et al., 1996).

A second candidate for the male component of the SI recognition system is the product of the SLA gene, which was originally identified in the $S_{2}$ haplotype where it is located just downstream of the $S L G_{2}$ gene (Boyes and Nasrallah, 1995). SLA encodes two complementary transcripts that accumulate specifically in anthers. One of the transcripts contains two small open reading frames (ORFs), and it has been proposed that a peptide encoded by SLA might interact, either directly or indirectly, with SLG and SRK to initiate the SI response (Boyes and Nasrallah, 1995). A second SLA allele was shown to be interrupted 
by a large insertion resembling a retrotransposon in a self-compatible Brassica napus line suggesting a correlation between the presence of a functional SLA gene and SI (Boyes and Nasrallah, 1995). SLA was not detected in a number of other $S$ haplotypes, and it has been suggested that this might be due to a high level of polymorphism between SLA alleles (Boyes and Nasrallah, 1995), as would be expected for a gene involved in the SI response.

In this study, we have characterized the SLA gene from the self-compatible $B$. oleracea line P57Sc and have shown that it also contains a large insertion resembling a retrotransposon. However, the structure of the SLA gene in a number of B. oleracea var botrytis lines, which carry the same $S$ haplotype as P57Sc but which exhibit different levels self-incompatibility, indicated that a functional SLA gene is not required for the SI response. 


\section{RESULTS}

\section{Cloning of $S L G$ and $S L A$ from a self-compatible B. oleracea line}

The cloning and characterization of a cDNA clone, denoted CG15, from the B. oleracea line P57Sc has been described previously (Gaude et al., 1993). Based on sequence similarity to previously characterized $S L G$ genes, CG15 was thought to correspond to the $S L G$ gene of the P57Sc line $\left(S L G_{S c}\right.$; Gaude et al., 1993).

In this study, a 880-bp BamHI fragment from the 5' end of the CG15 cDNA was used to probe $10^{6}$ plaque-forming units of a P57Sc genomic library. Subcloning and DNA sequence analysis of phages, which hybridized with the probe, revealed that they included one clone with $100 \%$ sequence identity to CG15 and a second clone, denoted CG514, that was highly similar but not identical to CG15. A 10.5-kb region of the latter clone was sequenced and shown to share extensive similarity with the SLG/SLA region of the $S_{2}$ haplotype (Figure 1). Based on this similarity, the two genes carried by the CG514 clone have been designated $S L G_{S c}$ and $S L A_{S c}$, and hence, the gene represented by CG15 is no longer denoted $S L G_{S c}$, as was originally proposed (Gaude et al., 1993). We now propose that CG15 corresponds to a second, $S$ locus-linked gene and may represent an alternative transcript of the $S R K_{S c}$ gene encoding a truncated form of SRK analogous to the eSRK protein identified in the $S_{3}$ haplotype (Giranton et al., 1995).

\section{Comparison of the $S L G$ and $S L A$ genes of the $S_{S c}$ and $S_{2}$ haplotypes}

Alignment of the SLG/SLA gene region of the $S$ locus of the P57Sc line $\left(S_{S c}\right)$ with the corresponding region of the $S_{2}$ haplotype showed that the two sequences are highly similar, 
apart from an insertion of $4826 \mathrm{bp}$ in $S L A_{S c}$ that is described in more detail below. $S L G_{2}$ has been shown to encode two alternative transcripts, which encode the soluble $\mathrm{SLG}_{2}$ protein and a membrane-anchored form of $\mathrm{SLG}_{2}, \mathrm{mSLG}_{2}$, respectively. The $S L G_{S c}$ gene possesses both of the exons present in the $S L G_{2}$ gene and, therefore, potentially encodes both SLG and mSLG proteins. The deduced amino acid sequences of $S L G_{S c}$ and $S L G_{2}$ share $98.2 \%$ similarity. In both the $S_{S c}$ and $S_{2}$ haplotypes, $S L A$ is located directly downstream of $S L G$ (Figure 1). Boyes and Nasrallah (1995) reported the sequences of cDNAs corresponding to two overlapping transcripts of the $S L A_{2}$ gene: a spliced transcript that was transcribed from the same strand as $S L G_{2}$ and that contains two ORFs and an unspliced transcript that was transcribed from the opposite strand. Sequences corresponding to both of these transcripts are present in $S L A_{S c}$ and exhibit $99.6 \%$ nucleotide similarity with the spliced and unspliced transcripts of $S L A_{2}$ (Figure 1). The two ORFs in the spliced transcript are identical between the two alleles, except that the eighth codon of ORF2 is an alanine codon (GCG) in $S L A_{2}$ and an arginine codon (CGC) in $S L A_{S c}$.

The 4826-bp insertion in $S L A_{S c}$ contains all the structural features of a retrotransposon (Figure 2; reviewed by Grandbastien, 1992). The insertion contains two long terminal repeats (LTRs) that differ by 7-bp at a single position $32 \mathrm{bp}$ from the $3^{\prime}$ end. The LTRs are flanked by a duplicated 6-bp sequence of the SLA gene resembling the direct repeats found at sites of transposon insertion. Between the two LTRs is a region of 4432 bp that is flanked by sequences resembling sites involved in priming reverse transcription of known retrotransposons (Figure 2A). These sites consist of an 11-bp region identical to the initiator methionine tRNA from Arabidopsis at the 5' end of the internal domain and a purine-rich sequence at the $3^{\prime}$ end.

The 4432-bp internal domain contains two long ORFs (Figure 2B). ORF1 is predicted to encode an 1132-amino acid polypeptide that includes regions similar to conserved motifs 
found in retransposons (Figure 2B): an RNA binding site involved in positioning the tRNA during the initiation of reverse-transcription; a protease domain implicated in the endoproteolytic cleavage of the polypeptide; an integrase region necessary for the integration of the retroelement in the host genome, and a reverse transcriptase domain. ORF2 is located $64 \mathrm{bp}$ downstream of the end of ORF1 and is predicted to encode a 253 amino acid protein with an RNaseH domain. Comparison of the sequence of this element, which we have named Melmoth (Maturin, 1820), with previously identified retrotransposons showed that it is most closely related to the $T y 1 /$ copia family of retroelements (Figure 2B).

A DNA fragment from within the Melmoth element (see Figure 1) was used to probe a DNA blot of restriction endonuclease digested genomic DNA of the B. oleracea var acephala and B. oleracea var botrytis (cauliflower) lines used in this study. The results indicated the presence of between one and three copies of the Melmoth sequence per haploid genome, depending on the genotype (data not shown). These results suggest that the Melmoth element is not replicating at a high frequency in the genome of the lines analyzed and it is possible that the element is an inactive member of the Tyl/copia family. However, low copy number does not necessarily indicate that an element is inactive; the maize retrotransposon $B s 1$, which is present at only two to three copies in the genome, is nonetheless active because it was isolated following a transposition event (Jin et al., 1989). On the other hand, the 7-bp difference between the two LTRs of Melmoth indicate that its insertion into SLA was not recent. The mechanism of transposition of yeast and insect retrotransposons has been well characterized and involves the formation of a terminally redundant, circular RNA molecule and, hence, the production of retrotransposons with identical LTRs. Assuming that Melmoth is mobilized by a similar mechanism, the difference between the two LTRs is likely to have been acquired subsequent to its insertion into the $S L A$ gene. It remains to be determined whether this difference prevents subsequent transpositions of this element. 
$S L G_{S c}$ and $S L A_{S c}$ are linked to the $S$ locus

To demonstrate that $S L G_{S c}$ and $S L A_{S c}$ are linked to the $S$ locus, we analyzed an $\mathrm{F}_{2}$ population of 23 plants resulting from a cross between the homozygous P57Sc line and a line homozygous for the $S_{3 a}$ haplotype. The segregation of the two $S$ haplotypes was followed by immunoblot analysis of stigma proteins separated by isoelectric focusing. A battery of antibodies raised against both class I and class II SLG proteins which had been developed in our Lyon laboratory for the purpose of determining the $S$ haplotype of Brassica plants (Delorme et al., 1995a), was used for this analysis. Segregation of $S L A_{S c}$ was followed by polymerase chain reaction (PCR) amplification of $S L A_{S c}$ genomic DNA sequences using oligonucleotides OSLA1 and OSLA2 (see Figure 1 for the positions of the oligonucleotides relative to the $S L A$ sequence). Figure 3 shows that an $S L A_{S c}$ PCR product was only amplified from genomic DNA of $\mathrm{F}_{2}$ progeny carrying the $S_{S c}$ haplotype, indicating that $S L A_{S c}$ is linked to the $S$ locus in the P57Sc line.

\section{Analysis of self-compatible and self-incompatible B. oleracea var botrytis lines carrying transposon-mutated SLA genes}

To investigate further the role of SLA in SI, we analyzed a number of B. oleracea var botrytis lines also carrying class II $S$ haplotypes. The origins and SI phenotypes of the B. oleracea var botrytis lines are described in Table 1. Surprisingly, despite the fact that the different lines exhibited different levels of self-incompatibility, several lines of evidence indicate that all of the B. oleracea var botrytis lines carry the $S_{S c}$ haplotype. Monoclonal antibody (MAb) 15735-50, an antibody raised against a peptide corresponding to the $\mathrm{N}$ terminus of the SLG 
protein of the P57Sc line (Gaude et al., 1993), recognizes SLG proteins from a range of class II $S$ haplotypes, and closely related alleles can be distinguished based on the isoelectric points of the immunodetected proteins when separated by isoelectic focusing (Delorme et al., 1995a).

Figure 4A shows that the pattern of stigma glycoproteins detected by MAb 157-35-50 in extracts from seven $B$. oleracea var botrytis lines was highly similar to that of the P57Sc line but markedly different from that of the two lines carrying the closely related $S_{2}$ and $S_{5}$ haplotypes. This result suggested that all the $B$. oleracea var botrytis lines carry the $S_{S c}$ haplotype and this hypothesis was supported by DNA sequence analysis of PCR products from the B. oleracea var botrytis lines. A 1018-bp region corresponding to nucleotides 317 to 1335 of the CG15 cDNA and including regions identified as highly variable in SLG sequences (Nasrallah et al., 1987) was amplified using the PCR from genomic DNA of each of the B. oleracea var botrytis lines and cloned into a plasmid vector. The DNA sequences of all seven fragments were identical to the corresponding region of the CG15 cDNA from P57Sc (data not shown). In addition, in reciprocal genetic crosses, pollen of the $B$. oleracea var botrytis lines 11035 and 40443 was rejected by stigmas of the P57Sc line whereas pollen from P57Sc was compatible on stigmas of these two lines. These results indicate that the female part of the SI recognition system is functional in the P57Sc line, and that it recognizes pollen from the 11035 and 40443 lines as "self", i.e., as carrying the same $S$ haplotype. Taken together, these results indicate that the $B$. oleracea var botrytis lines used in this study carry the same $S$ haplotype as P57Sc.

Because the different $B$. oleracea var botrytis lines exhibited different levels of SI, we were interested in determining whether their $S L A$ genes would be interrupted by an insertion. Therefore, PCR amplification was carried out using oligonucleotides OSLA1, OSLA2, and OSLA3 (see Figure 1 for oligonucleotides). This combination of oligonucleotides was 
predicted to give a 798-bp PCR product in the presence of an SLA gene carrying the putative retrotransposon and a 612-bp PCR product if an uninterrupted SLA gene was provided as template. PCR amplification from genomic DNA of each of the B. oleracea var botrytis lines resulted in a product of 798-bp indicating that all seven carried an SLA gene interrupted by the putative retrotransposon (Figure 4B). The fact that the size of the PCR products amplified from DNA of the seven B. oleracea var botrytis lines was the same as that amplified from DNA of the P57Sc line (Figure 4B) indicated that the putative retrotransposon was inserted at the same position in the SLA genes of all these lines. The smaller, 612-bp amplification product obtained with $S_{2}$ DNA is consistent with the absence of an insert in the $S L A_{2}$ gene. No amplification product was obtained from DNA of the $S_{5}$ haplotype. This is consistent with the observation of Boyes and Nasrallah (1995) that this haplotype did not contain sequences which hybridised to an $S L A_{2}$ probe .

The experiment shown in Figure 4B demonstrated that all of the B. oleracea var botrytis lines, including the strongly self-incompatible lines 11035, 24062, and 41865, carried an interrupted SLA gene. It was therefore important to determine whether the insertion interfered with expression of $S L A$. In the $S_{2}$ haplotype, which contains a wild-type $S L A$ gene lacking an inserted retrotransposon sequence, Boyes and Nasrallah (1995) have shown that the 1.56-kb, spliced $S L A$ transcript accumulates in young anthers, with a maximum abundance in 7- to 9-mm long flower buds (which correspond to the bicellular and tricellular stages of microspore development) and that the $2.17-\mathrm{kb}$ antisense unspliced transcript accumulates later and is most abundant when the flower buds are more than 9-mm long (anthers containing tricellular microspores and mature pollen). The increase in abundance of the unspliced transcript is concurrent with a decrease in abundance of the spliced transcript. The site of insertion of the putative retrotransposon in $S L A_{S c}$ corresponds to a region that is transcribed in both directions in $S L A_{2}$. The corresponding site in $S L A_{2}$ is 198 bp from the $3^{\prime}$ 
end of the unspliced transcript and $492 \mathrm{bp}$ from the $5^{\prime}$ end of the spliced transcript. An insertion in this region would be expected to perturb synthesis of both of the transcripts, particularly of the spliced transcript that is thought to encode a peptide product (Boyes and Nasrallah, 1995).

We used RNA gel blot analysis to assay for the presence of SLA transcripts in different Brassica lines (Figure 5). Poly $(\mathrm{A})^{+}$RNA was extracted from anthers at developmental stages corresponding to the stages when either the spliced or the unspliced transcripts were most abundant in the $S_{2}$ haplotype studied by Boyes and Nasrallah (1995). An RNA gel blot was probed with a DNA fragment from a region of the $S L A_{S c}$ gene corresponding to a region that is transcribed in both directions in $S L A_{2}$. Bands corresponding to both the spliced and unspliced transcripts were detected in anther poly(A) ${ }^{+}$RNA from an $S_{2}$ homozygous line but neither SLA transcript was detected in anthers from either P57Sc or the self-incompatible $B$. oleracea var botrytis line 11035. The two SLA transcripts were, therefore, not detected in lines carrying an $S L A_{S c}$ allele with an inserted Melmoth element, indicating that the presence of this element prevents expression of the SLA gene. The absence of detectable transcripts in the self-incompatible B. oleracea var botrytis line 11035 indicates that SLA function is not required for the SI response.

\section{$S L A$ is a single copy, homozygous gene in the Brassica lines used in this study}

Other possible explanations for the self-incompatible phenotype of some of the B. oleracea var botrytis lines are either that they were heterozygous at the SLA locus or that SLA is a member of a functionally redundant gene family, with only one active member being required for the SI response. All seven of the B. oleracea var botrytis plants were derived from lineages that had undergone several generations of self-fertilization (Table 1); therefore they 
were highly likely to be homozygous at the $S L A$ locus. To confirm this, the seven B. oleracea var botrytis plants were self-fertilized and the segregation of SLA was followed in the progeny. Genomic DNA was prepared from the progeny, and the SLA gene structure was analyzed by PCR amplification using oligonucleotides OSLA1, OSLA2, and OSLA3. All of the progeny analyzed (a total of 117 representing between 10 and 29 progeny per line depending on the line) carried the SLA allele interrupted by an insertion (Table 2). These data provide strong evidence that the parental plants were homozygous at the SLA locus, for example a probability of homozygosity of greater than $99 \%$ was calculated for the three strongly self-incompatible B. o. var botrytis lines 11035, 24062, and 41865 (assuming a normal Mendelian segregation of SLA alleles).

Moreover, there is no evidence that $S L A$ is part of a gene family. Boyes and Nasrallah (1995) showed that SLA is a single-copy gene in the genome of a line homozygous for the $S_{2}$ haplotype. We have confirmed this result and have shown that SLA is also single-copy gene in P57Sc and the seven B. oleracea var botrytis lines. Figure 6 shows that a DNA probe derived from the SLA gene (see Figure 1) detected a single hybridizing fragment per lane at low stringency in a gel blot of restriction endonuclease digested genomic DNA from the P57Sc line, the $S_{2}$ homozygous line, and the seven $B$. oleracea var botrytis lines. No hybridizing fragment was detected in DNA of the class I $S_{3}$ haplotype. The SLA probe detected a 3.8-kb fragment in HindIII-digested genomic DNA from P57Sc and all seven $B$. oleracea var botrytis lines, again supporting the hypothesis that these lines carry the same $S$ haplotype.

\section{DISCUSSION}


In this study, we have characterized an allele of the $S$ locus-linked gene $S L A$ from the $S_{S c}$ haplotype carried by the $B$. oleracea var acephala line P57Sc. This allele, $S L A_{S c}$, was shown to be highly similar to the $S L A_{2}$ allele from the $S_{2}$ haplotype (Boyes and Nasrallah, 1995), except that it contained a large insertion of 4826-bp that resembled retrotransposons of the Ty1/copia family (Figures 1 and 2). Analysis of the structure of $S L A_{S c}$ provided conflicting evidence concerning the role of SLA in the self-incompatible response. Plants homozygous for the $S_{S c}$ locus have been shown to exhibit a self-compatible phenotype (Gaude et al., 1993). The presence of an insertion in the $S L A$ gene of this haplotype therefore provided a correlation between the self-compatible phenotype and the presence of a mutated SLA gene. However, the high degree of similarity between $S L A_{S c}$ and $S L A_{2}$ is not consistent with the proposed highly polymorphic nature of SLA (Boyes and Nasrallah, 1995). Genes involved in the recognition step of the SI response are expected to be highly polymorphic, with a different allele existing for each $S$ haplotype. Boyes and Nasrallah (1995) proposed that SLA was not detected in haplotypes other than $S_{2}$ (and the closely related B. napus haplotype $S_{2 W}$ ) because a high level of polymorphism prevented cross-hybridization of probes. However, comparison of the $S_{2}$ and $S_{S c}$ haplotypes showed that the nearby $S L G$ genes had diverged to a greater extent (98.6\% nucleotide similarity) than the two SLA genes (99.6\% nucleotide similarity) with SLA exhibiting a very low level of polymorphism (Figure 1).

To further investigate the role of SLA in the SI response, we analyzed a number of $B$. oleracea var botrytis lines carrying $S_{S c}$ or a very closely related $S$ haplotype but exhibiting different degrees of self-fertility (Figures 3 and 4; Table 1). All of these lines analyzed carried a single-copy homozygous $S L A$ gene that was interrupted by an insertion (Figure 6). No SLA transcripts were detected in anthers of B. oleracea var botrytis line 11035, which nonetheless exhibited a strong self-incompatible response. These data strongly indicate that a functional SLA gene is not required for the SI response in B. oleracea. 
The $S$ locus is predicted to encode both the male and female components of the SI recognition system, and several studies have been initiated recently to identify the gene encoding the pollen component by analysis of the regions flanking the $S L G$ and $S R K$ genes of different $S$ haplotypes. Boyes et al. (1997) analyzed the regions downstream of the $S L G_{8}$ and $S L G_{13}$ alleles of $B$. campestris and B. oleracea, respectively, for sequences that exhibited haplotype specificity and anther-specific expression in a search for functional homologs of $S L A_{2}$. No such sequences were found. This supports our hypothesis, based on the high degree of similarity between $S L A_{2}$ and $S L A_{S c}$, that $S L A$ exhibits a low level of polymorphism and indicates that $S L A$ is not present at the $S$ locus in all $S$ haplotypes.

In the same study, Boyes et al. (1997) identified two novel genes, 298 and 299, downstream of $S L G_{8}$ in $B$. campestris. These genes were not characterized in detail, but the fact that both genes are expressed in leaves as well as floral organs and that gene-specific probes hybridize equally strongly with DNA from lines carrying the $S_{8}, S_{2}$ or $S_{13}$ haplotypes argues against these genes being involved in determining the specificity of the SI response. Similarly, Yu et al. (1996) characterized two novel genes, $S L L_{1}$ and $S L L_{2}$, located between $S L G$ and $S R K$ in both the 910 and A10 $S$ haplotypes of B. napus. The DNA sequence of $S L L_{1}$, and probably also of $S L L_{2}$, is $100 \%$ conserved between the two $S$ haplotypes, indicating that neither gene is involved in determining the specificity of the SI response (although one or both of these genes may play a more general role in SI). Taken together, the analyses of SLA, 298, 299, $S L L_{1}$, and $S L L_{2}$ have revealed that genes exhibiting a low level of polymorphism are present at the $S$ locus close to the high polymorphic $S L G$ and $S R K$ genes and that, at least in the case of $S L A$, localization at the $S$ locus does not necessarily imply a role in the SI response.

Based on the low level of polymorphism of $S L L_{1}$ and $S L L_{2}$ compared with $S L G$ and $S R K$, Yu et al. (1996) have proposed a model in which the former were inserted into the $S$ 
locus after the divergence of the different $S$ haplotypes. $S L L_{1}$ and $S L L_{2}$ would then have spread from one $S$ haplotype to another by homologous recombination. The fact that SLA is present in the two closely related $S_{2}$ and $S_{S c}$ haplotypes but was not detected in the $S_{5}, S_{3}$, or $S_{3 a}$ haplotype (Boyes and Nasrallah, 1995 and this work) indicates that SLA may similarly have been inserted into the $S$ locus during the divergence of the different $S$ haplotypes perhaps into the common ancestor of $S_{S c}$ and $S_{2}$. We cannot, however, rule out the possibility that $S L A$ has been deleted from the other $S$ haplotypes during evolution or that it has been transferred from $S_{2}$ to $S_{S c}$ or visa versa by a recombination event. The insertion of Melmoth into $S L A$ presumably occurred after the divergence of the different $S$ haplotypes in Brassica but before the divergence of B. oleracea and B. napus.

Interestingly, a retrotransposon-like sequence has also been identified at the $S$ locus in Nicotiana alata (Royo et al., 1996). N. alata possesses a gametophytic SI system in which pollen rejection is mediated by stigmatic $S$ RNases. There is no known sequence homology between the $S$ locus genes of gametophytic and sporophytic SI systems. However, in both cases, sequence polymorphism between alleles is known to extend for several kilobases into the regions surrounding the $S$ locus genes, indicating that there has been extensive rearrangements in these regions of the respective genomes (Clark et al., 1990; Coleman and Kao, 1992; Yu et al., 1996; Boyes et al., 1997). It will be interesting to determine whether the presence of putative retrotransposons at two different $S$ loci is coincidental or whether their presence is related in some way to the unusual structure of $S$ loci.

Boyes and Nasrallah (1995) have described an SLA allele $\left(S L A_{2 w}\right)$ from B. napus var Westar that, like $S L A_{S c}$, contains a large insertion within the transcribed part of the gene. Although no sequence data was provided, the description of this allele indicates that it strongly resembles the $S L A_{S c}$ allele described above. The B. napus allele is greater than $99 \%$ identical to $S L A_{2}$, apart from a large insert of 4827 bp ( $S L A_{S c}$ contains an insert of $4826 \mathrm{bp}$ ). 
In particular, the two ORFs are highly conserved between $S L A_{2 w}$ and $S L A_{S c}$, with only one amino acid difference between each ORF. No SLA transcripts were detected in B. napus plants carrying $S L A_{2 w}$ by RNA blot analysis (Boyes and Nasrallah, 1995).

Evidence has been available for some time indicating that the $S_{S c}$ haplotype is present in the genome of B. napus var Westar. Robert et al. (1994) have described a full-length cDNA from this variety which has a nucleotide sequence $100 \%$ identical to that of the CG15 cDNA isolated from the B. oleracea P57Sc line (Gaude et al., 1993). Robert et al. (1994) named the cDNA $S L G_{W S 2}$; however, based on our analysis of the P57Sc line, this cDNA, like CG15, most likely corresponds to an alternative transcript of SRK (Giranton et al., 1995 and see above). Moreover, we have analyzed 10 self-compatible, commercially available varieties of B. napus by sequencing of PCR-amplified fragments and have shown that all 10 carry sequences identical to the CG15 cDNA indicating that $S_{S c}$ or a very closely related $S$ haplotype is common in commercial varieties of B. napus (V. Delorme and J. M. Cock, unpublished data). Based on these data, it seems likely that the mutated SLA gene identified by Boyes and Nasrallah (1995) is part of an $S$ haplotype closely related to the $S_{S c}$ haplotype in B. napus and, for the reasons described above, it would, therefore, seem unlikely that the selfcompatible nature of Westar is related to the presence of a nonfunctional SLA gene. However, the fact that an $S$ haplotype closely related to the $S_{S c}$ is widespread in self-compatible $B$. napus is interesting considering that this haplotype is associated with a self-compatible phenotype in some lines of $B$. oleracea (Gaude et al, 1993; this study). In addition, the $S_{S c}$ haplotype carried by the $B$. oleracea P57Sc line is particularly interesting in that genetic crosses indicate that the male component of the SI response is defective. Further work will be aimed at determining the molecular basis of the self-compatible phenotype associated with this haplotype. 


\section{METHODS}

\section{Plant Material, Genetic Crosses, and Determination of Incompatibility Phenotype}

The self-compatible B. oleracea var acephala line P57Sc has been described previously (Gaude et al., 1993). An $\mathrm{F}_{2}$ population obtained by self-fertilization of an $S_{3 a} / S_{S c} \mathrm{~F}_{1}$ hybrid (the parental lines are described in Delorme et al., 1995a, and Gaude et al., 1993, respectively) was used to demonstrate linkage of $S L A$ with the $S$ locus. The incompatibility phenotypes of the $\mathrm{F}_{2}$ progeny were determined by self-pollination and by crosses to tester plants using previously described procedures (Delorme et al., 1995b). Of the seven $B$. oleracea var botrytis used in this study, five were commercial and open-pollinated varieties, either Lecerf (41865) or Automne malouin tardif (11035, 17144, 40443, and 14548), and two were non-commercial and open-pollinated varieties (24062 and 22495). Incompatibility phenotype was determined by aniline blue staining of pollinated pistils as previously described (Ruffio-Châble et al., 1997).

\section{Genomic DNA Cloning and DNA Sequencing}

The $S L G_{S c} / S L A_{S c}$ gene region was isolated from a genomic library constructed with DNA of the P57Sc line. The construction and screening of the library were as described previously (Delorme et al., 1995b). Restriction endonuclease digested fragments of isolated $\lambda$ clones were subcloned into pBluescript II SK ${ }^{+}$(Stratagene, La Jolla, CA) and sequenced using the dideoxynucleotide chain termination method (Sanger et al., 1977) either on an automatic sequencer (Applied Biosystems, Foster City, CA) or using a T7 DNA polymerase sequencing kit (Pharmacia Biotech, Uppsala, Sweden). Sequence data was analysed using Lasergene 
sequence analysis software (DNASTAR, London, UK). The results of comparisons are expressed as percentage similarity, which is calculated as follows: 100 times the number of matched amino acids, divided by the sum of the length in amino acids of the aligned region plus the number of gaps introduced to optimise the alignment.

\section{Protein Extraction, Electrophoretic Analysis, and Immunodetection of Proteins}

Protein extraction, separation of proteins by isoelectric focusing or SDS-PAGE, electrotransfer onto nitrocellulose membranes and detection of antigen with antibodies were as described previously (Gaude et al., 1991, 1993). Monoclonal antibody (MAb) 157-35-50 has been described previously (Giranton et al., 1995).

\section{DNA Extraction, Polymerase Chain Reaction Amplification and DNA Gel Blots}

Genomic DNA for DNA gel blot analysis and polymerase chain reaction (PCR) experiments was extracted as follows. Two centimeter square pieces of leaf were ground to a powder in liquid nitrogen and incubated at $65^{\circ} \mathrm{C}$ in $500 \mu \mathrm{L}$ of extraction buffer (extraction buffer is 0.1 M Tris, pH 8, $50 \mathrm{mM}$ EDTA, $100 \mathrm{mM} \mathrm{NaCl}, 1 \%$ SDS, and $9 \mu \mathrm{M}$ 2-mercaptoethanol). After the addition of $165 \mu \mathrm{L}$ of $3 \mathrm{M}$ potassium acetate and $500 \mu \mathrm{L}$ of a phenol-chloroform mixture (1:1), the samples were agitated for $5 \mathrm{~min}$, incubated for $10 \mathrm{~min}$ on ice, and centrifuged for 15 min at $18000 \mathrm{~g}$ at $4^{\circ} \mathrm{C}$. The aqueous fraction was mixed with $750 \mu \mathrm{L}$ of isopropanol and incubated for $2 \mathrm{hr}$ at room temperature. Genomic DNA was recuperated by centrifugation and washed with $70 \%$ ethanol before being resuspended in $100 \mu \mathrm{L}$ of water.

When the DNA was prepared for PCR analysis, samples of tissue from a Brassica plant, which did not contain the template sequence for the PCR (leaves of a B. oleracea var 
acephala plant homozygous for the $S_{3}$ haplotype) were harvested after every fifth test sample. These intercalated control samples underwent the same extraction and PCR amplification steps as the test samples to check that there was no cross-contamination between samples. The oligonucleotides used for PCR amplification were: OSLA1 (5'GAAGCCGACCCGTTTGGA-3'), OSLA2 (5'-GAGAGAAGGCGGAGGTGA-3'), and OSLA3 (5'-GTCCTGGCGTTTGAGAGC-3'), which correspond to $S L A_{S c}$ sequences; and SG2 (5'-GGCCTGCAGCAGCATTCAATCTGAC-3') and SG22 TGGAACCCTCAAAATCT-3'), which correspond to CG15 (Gaude et al., 1993).

For DNA blot analysis, $10 \mu \mathrm{g}$ of genomic DNA was digested with the HindIII, fractionated on $0.8 \%$ agarose gels, and transferred to nylon membranes (Hybond $\mathrm{N}^{+}$; Amersham) under alkaline conditions. A 1260-bp EcoRV-BamHI restriction fragment of SLA and a 860-bp EcoRV restriction fragment of the insertion in SLA were used as probes for SLA and the Melmoth element respectively. The probes are positioned with respect to the $S L G / S L A$ region of the $S_{S c}$ haplotype in Figure 1. Radiolabeled DNA probes were prepared using a random priming DNA labelling kit (Boehringer Mannheim). Filters were prehybridized and hybridized at $42^{\circ} \mathrm{C}$ in $50 \%$ formamide, $6 x \mathrm{SSC}$ (1xSSC is $0.15 \mathrm{M} \mathrm{NaCl}, 0.015 \mathrm{M}$ sodium citrate), $0.5 \%$ SDS, $0.1 \%$ ficoll, $0.1 \%$ PVP, $0.1 \%$ BSA, and herring sperm DNA (100 $\mu \mathrm{g} \mathrm{mL}-$ 1). Identical results were obtained after washing at either low (30 min in 6xSSC, $0.1 \%$ SDS at $50^{\circ} \mathrm{C}$ ) or high stringency $\left(30 \mathrm{~min}\right.$ in $0.1 \mathrm{xSSC}, 0.1 \% \mathrm{SDS}$ at $\left.50^{\circ} \mathrm{C}\right)$.

\section{RNA Gel Blot Analysis}

Developmental stages of anthers were determined by fluorescence microscopy observation of 4',6-diamidino-2-phenylindole-stained microspores and approximately correlated with bud length. Immature anthers containing unicellular and bicellular microspores were dissected 
from either 5 to $9 \mathrm{~mm}$ flower buds for B. oleracea var acephala plants or from 3 to $6 \mathrm{~mm}$ flower buds for B. oleracea var botrytis plants, which have proportionally smaller flowers. Similarly, anthers containing tricellular microspores were dissected from $9 \mathrm{~mm}$ or $6 \mathrm{~mm}$ flower buds of B. oleracea var acephala or B. oleracea var botrytis plants, respectively. Total RNA extraction was as described by Cock et al. (1997). Poly(A) ${ }^{+}$RNA was purified by hybridization with magnetic beads coated with oligo(dT) ${ }_{25}$ (Novagen, Inc., Madison, WI), and $16 \mu \mathrm{g}$ of poly $(\mathrm{A})^{+}$RNA was separated in each lane of a formaldehyde gel before being transferred to a nylon filter for hybridization. Preparation of radiolabelled probes and hybridisation conditions were as described by Pastuglia et al. (1997).

\section{ACKNOWLEDGMENTS}

We thank Marie-Angèle Grandbastien (INRA, Versailles, France) for her comments on the manuscript, Dr. Mikail E. Nasrallah (Cornell University, Ithaca, NY) for the actin probe, and Richard Blanc, Hervé Leyral, Fabienne Deguerry, Monique Estienne and Anne-Marie Thierry for technical assistance. M.P., V.R., and J.M.C. are members of the Institut National de la Recherche Agronomique, and T.G. is a member of the Centre National de la Recherche Scientifique. This work was supported jointly by the INRA and the CNRS.

\section{REFERENCES}

Akama, K., and Tanifuji, S. (1989). Nucleotide sequence of a methionine initiator tRNA gene of Arabidopsis thaliana. Plant Mol. Biol. 13, 599-600.

Becraft, P.W., Stinard, P.S., and McCarty, D.R. (1996). CRINKLY4: a TNFR-like receptor kinase involved in maize epidermal differentiation. Science 273, 1406-1409. 
Boyes, D.C. and Nasrallah, J.B. (1993). Physical Linkage of the $S L G$ and $S R K$ genes at the self-incompatibility locus of Brassica oleracea. Mol. Gen. Genet. 236, 369-373.

Boyes, D.C., and Nasrallah, J.B. (1995). An anther-specific gene encoded by an $S$ locus haplotype of Brassica produces complementary and differentially regulated transcripts. Plant Cell 7, 1283-1294.

Boyes, D.C., Nasrallah, M.E., Vrebalov, J., and Nasrallah, J.B. (1997). The selfincompatibility $(S)$ haplotypes of Brassica contain highly divergent and rearranged sequences of ancient origin. Plant Cell 9, 237-247.

Braun, D.M., and Walker, J.C. (1996). Plant transmembrane receptors: new pieces in the signalling puzzle. Trends Biochem. Sci. 21, 70-73.

Camirand, A., and Brisson, N. (1990). The complete nucleotide sequence of the Tst1 retrotransposon of potato. Nucleic Acids Res. 18, 4929.

Clark, K.R., Okuley, J.J., Collins, P.D., and Sims, T.L. (1990). Sequence variability and developmental expression of S-alleles in self-incompatible and pseudo-self-compatible petunia. Plant Cell 2, 815-826.

Cock, J.M., Swarup, R., and Dumas, C. (1997). Natural antisense transcripts of the $S$ locus receptor kinase gene and related sequences in Brassica oleracea. Mol. Gen. Genet., $255,514-524$.

Coleman, C.E., and Kao, T.-h. (1992). The flanking regions of two Petunia inflata S alleles are heterogeneous and contain repetitive sequences. Plant Mol. Biol., 18, 725-737.

Delorme, V., Gaude, T. Heizmann, P., and Dumas, C. (1995a). Use of immunochemical and SSCP analyses to test homozygosity at the $S$ locus of Brassica oleracea genotypes. Mol. Breeding 1, 237-244.

Delorme, V., Giranton, J.L., Hatzfeld, Y., Friry, A., Heizmann, P., Ariza, M.J., Dumas, C., Gaude, T., and Cock, J.M. (1995b). Characterization of the $S$ locus genes, SLG 
and $S R K$, of the Brassica $S_{3}$ haplotype: identification of a membrane-localized protein encoded by the $S$ locus receptor kinase gene. Plant J. 7, 429-440.

Doughty, J., Hedderson, F., McCubbin, A., and Dickinson, H. (1993). Interaction between a coating-borne peptide of the Brassica pollen grain and stigmatic- $S$ (selfincompatibility)-locus-specific glycoproteins. Proc. Natl. Acad. Sci. USA 90, 467-471.

Gaude, T., Denoroy, L., and Dumas, C. (1991). Use of a fast protein electrophoretic purification procedure for $N$-terminal sequence analysis to identify $S$-locus related proteins in stigmas of Brassica oleracea. Electrophoresis 12, 646-653.

Gaude, T., Friry, A., Heizmann, P., Mariac, C., Rougier, M., Fobis, I., and Dumas, C. (1993) Expression of a self-incompatibility gene in a self-compatible line of Brassica oleracea. Plant Cell, 5, 75-86.

Giranton, J.-L., Ariza, M.J., Dumas, C., Cock, J.M., and Gaude, T. (1995). The $S$ locus receptor kinase gene encodes a soluble glycoprotein corresponding to the SRK extracellular domain in Brassica oleracea. Plant J. 8, 101-108.

Goring, D.R., and Rothstein, S.J. (1992). The $S$-locus receptor kinase gene in a selfincompatible Brassica napus line encodes a functional serine/threonine kinase. Plant Cell 4, 1273-1281.

Goring, D.R., Glavin, T.L., Schafer, U., and Rothstein, S.J. (1993). An $S$ receptor kinase gene in self-compatible Brassica napus has a 1 bp deletion. Plant Cell 5, 531-539.

Grandbastien, M.-A. (1992). Retroelements in higher plants. Trends Genet. 8, 103-108.

Hervé, C., Dabos, P., Galaud, J.-P., Rougé, P., and Lescure, B. (1996). Characterization of an Arabidopsis thaliana gene that defines a new class of putative plant receptor kinases with an extracellular lectin-like domain. J. Mol. Biol. 258, 778-788.

Jin, Y.-K., and Bennetzen, J.L. (1989). Structure and coding properties of Bsl, a maize retrovirus-like transposon. Proc. Natl. Acad. Sci. USA 86, 6235-6239. 
Jakobsen K.S., Breivold E., and Hornes E. (1990). Purification of mRNA directly from crude plant tissues in 15 minutes using magnetic oligo dT microspheres. Nucleic Acids Res. 18, 3669.

Maturin, C. (1820). Melmoth the Wanderer: a Tale (London: Archibald Constable and Company).

McCubbin, A.G., and Kao, T.-h. (1996). Molecular mechanisms of self-incompatibility. Curr. Opin. Biotech. 7, 150-154.

Mount, S.M., and Rubin, G.M. (1985). Complete nucleotide sequence of the Drosophila transposable element copia: homology between copia and retroviral proteins. Mol. Cell. Biol. 5, 1630-1638.

Nasrallah, J.B., Kao, T.-H., Goldberg, M.L., and Nasrallah, M.E. (1985). A cDNA clone encoding an S-locus specific glycoprotein from Brassica oleracea. Nature 318, 263267.

Nasrallah, J.B., Kao, T.-H., Chen, C.-H., Goldberg, M.L., and Nasrallah, M.E. (1987). Amino-acid sequence of glycoproteins encoded by three alleles of the $S$-locus of Brassica oleracea. Nature 326, 617-619.

Nasrallah, J.B., Rundle, S.J., and Nasrallah, M.E. (1994a). Genetic evidence for the requirement of the Brassica $S$-locus receptor kinase gene in the self-incompatibility response. Plant J. 5, 373-384.

Nasrallah, J.B., Stein, J.C., Kandasamy, M.K., and Nasrallah, M.E. (1994b). Signalling the arrest of pollen tube development in self-incompatible plants. Science 266, 15051508.

Ockendon, D.J. (1974). Distribution of self-incompatibility alleles and breeding structure of open-pollinated cultivars of Brussel sprouts. Heredity 33, 159-171. 
Ockendon, D.J. (1982). An S-allele survey of cabbage (Brassica oleracea var capitata). Euphytica 31, 325-331.

Pastuglia, M., Roby, D., Dumas, C., and Cock, J.M. (1997) Rapid induction by wounding and bacterial infection of an $S$ gene family receptor-like kinase gene in Brassica oleracea. Plant Cell 9, 49-60.

Ruffio-Châble, V., Hervé, Y., Dumas, C., and Gaude, T. (1997). Distribution of $S$ haplotypes and its relationship with self-incompatibility in Brassica oleracea. Part 1. In inbred lines of cauliflower (B. oleracea var 'botrytis'). Theor. Appl. Genet. 94, 338-346.

Robert, L.S., Allard, S., Franklin, T.M., and Trick, M. (1994). Sequence and expression of endogenous $S$-locus glycoprotein genes in self-compatible Brassica napus. Mol. Gen. Genet. 242, 209-216.

Royo, J., Nass, N., Matton, D.P., Okamoto, S., Clarke, A.E., and Newbigin, E. (1997). A retrotransposon-like sequence linked to the S-locus of Nicotiana alata is expressed in styles in response to touch. Mol. Gen. Genet. 250, 180-188.

Sanger, F., Nicklen, S., and Coulson, A.R. (1977). DNA sequencing with chain termination inhibitors. Proc. Natl. Acad. Sci. USA 74, 5463-5467.

Sato, T., Thorness, M.K., Kandasamy, M.K., Nishio, T., Hirai, M., Nasrallah, J.B., and Nasrallah, M.E. (1991). Activity of an $S$ locus gene promoter in pistils and anthers of transgenic Brassica. Plant Cell 3, 867-876.

Schulze-Muthe, P., Irmler, S., Schröder, G., and Schröder, J. (1996). Novel type of receptor-like protein kinase from a higher plant (Catharanthus roseus). J. Biol. Chem. 271, 26684-26689.

Stanchev, B.S., Doughty, J., Scutt, C.P., Dickinson, H., and Croy, R.R. (1996). Cloning of PCP1, a member of a family of pollen coat protein (PCP) genes from Brassica oleracea 
encoding novel cysteine-rich proteins involved in pollen-stigma interactions. Plant J. 10, 303-313.

Stein, J.C., Howlett, B., Boyes, D.C., Nasrallah, M.E., and Nasrallah, J.B. (1991). Molecular cloning of a putative receptor protein kinase gene encoded at the selfincompatibility locus of Brassica oleracea. Proc. Natl. Acad. Sci. USA 88, 8816-8820.

Stein, J.C., Dixit, R., Nasrallah, M.E., and Nasrallah, J.B. (1996). SRK, the stigmaspecific $S$ locus receptor kinase of Brassica, is targeted to the plasma membrane in transgenic tobacco. Plant Cell 8, 429-445.

Swarup, R., Dumas, D., and Cock, J.M. (1996). A new class of receptor-like protein kinase gene from Arabidopsis thaliana possessing a domain with similarity to plant lectin genes. Plant Physiol. 111, 347.

Voytas, D.F., and Ausubel, F.M. (1988). A copia-like transposable element family in Arabidopsis thaliana. Nature 336, 242-244.

Wang, X., Zafian, P., Choudhary, M., and Lawton, M. (1996). The PR5K receptor protein kinase from Arabidopsis thaliana is structurally related to a family of plant defense proteins. Proc. Natl. Acad. Sci. USA 93, 2598-2602.

Yu K., Schafer U., Glavin T.L., Goring D.R., and Rothstein S.J. (1996). Molecular characterization of the $S$ locus in two self-incompatible Brassica napus lines. Plant Cell 8, 2369-2380. 


\section{FIGURE LEGENDS}

Figure 1. Structure of the $S L G / S L A$ gene region in the self-compatible $B$. oleracea line P57Sc carrying the $S_{S c}$ haplotype.

Sequences corresponding to transcribed regions in the $S_{2}$ haplotype are shown as half-arrows, above the line if the transcript runs from left to right and below the line if the transcript runs from right to left. The open box corresponds to the region of the $S_{S c}$ haplotype that has been sequenced in this study. Nucleotides differing from the corresponding region of the $S_{2}$ haplotype are indicated by a vertical line in the box. A 4826-bp insert found in $S L A_{S c}$ but absent from $S L A_{2}$ is marked by a triangle. Arrowheads mark the positions of three oligonucleotides, OSLA1, OSLA2, and OSLA3 (represented by 1, 2 and 3 respectively). An $S L A$-specific DNA probe (SLA probe) and a probe for the 4826-bp element inserted in the SLA gene (Melmoth probe) are shown as hatched bars. H, HindIII; R, EcoRI; B, BamHI; S, SalI; Xb, XbaI.

The EMBL accession number for the $S L G_{S c} / S L A_{S c}$ sequence is Y12321.

Figure 2. Sequence similarity between the Melmoth element inserted in $S L A_{S c}$ and retrotransposons of the Ty1/copia family.

(A) Schematic representation of the Melmoth element that interrupts $S L A$ in the $S_{S c}$ haplotype. The diagram shows details of the sequences at the borders of the different domains and compares the sizes of the domains with the corresponding regions of retrotransposons of the Ty1/copia family. Dotted lines indicate the positions of the different domains and sequences relative to the diagram. The lengths of the internal domains in base pairs and the lengths of 
the open reading frames within this region in amino acid residues (aa) are indicated for each element. A 6-bp duplication flanking the Melmoth element and resembling the direct repeats (DR) found at the integration sites of transposons is underlined. Conserved sequences corresponding to priming sites for initiation of reverse transcription are shown. These consist of an 11-bp region adjacent to the left LTR, which is identical to the $3^{\prime}$ end of the tRNA initiator $\left(\mathrm{tRNA}_{\mathrm{i}}\right.$ ) of Arabidopsis (Akama and Tanifuji, 1989) and a purine-rich sequence at the $5^{\prime}$ of the right LTR (underlined). Triangles, closed boxes and the open box represent direct repeats, LTRs and the internal domain respectively.

(B) Comparison of the organization of ORFs in the Melmoth element with those of the copia, Ta1-3, and Tst1 retrotransposons. The positions of conserved regions corresponding to the RNA binding site (RBS), the protease site (Prot), the endonuclease region (Endo), the reverse transcriptase region (RT), and the RNase $\mathrm{H}$ region are indicated by shaded boxes. Percent amino acid similarity of the copia, Tst1 and Tal-3 sequences compared with Melmoth for the different domains is indicated. Blocks of conserved sequence within each region are shown with invariant amino acids boxed. Their position relative to the diagram is indicated with dotted lines. Black boxes represent LTRs. copia is from Drosophila (Mount and Rubin, 1985), Tst1 is from potato (Camirand and Brisson, 1990) and Tal-3 is from Arabidopsis (Voytas and Ausubel, 1988).

Figure 3. $S L A_{S c}$ is genetically linked to the $S$ locus.

DNA was extracted from two parental plants, P57Sc $\left(S_{S c}\right)$ and a plant homozygous for the $S_{3 a}$ haplotype $\left(S_{3 a}\right)$, and from $23 \mathrm{~F}_{2}$ progeny $\left(\mathrm{F}_{2}\right)$ descended from the two parental plants. PCR amplification was carried out using oligonucleotides OSLA1 and OSLA2 (see Figure 1), and the PCR products were separated on an agarose gel and stained with ethidium bromide. The 
number at left indicates the length of the PCR products in base pairs. Segregation of the two $S$ haplotypes in the $F_{2}$ population was followed by immunoblot analysis of stigmatic SLG proteins separated by isoelectric focusing (Delorme et al., 1995a), and the $S$ haplotypes carried by the different progeny are indicated below the lanes. 
Figure 4. Comparison of $B$. oleracea var botrytis lines with B. oleracea var acephala lines carrying different $S$ haplotypes.

(A) Immunoblot analysis of proteins in stigma extracts of different lines of B. oleracea var acephala and B. oleracea var botrytis. Proteins were separated by IEF and probed with MAb 157-35-50 as the primary antibody. Stigmas were from B. oleracea var acephala lines homozygous for the $S_{5}, S_{2}$, and $S_{S c}$ (the P57Sc line) haplotypes and from the B. oleracea var botrytis lines $41865,24062,11035,17144,40443$, and 22495. The positions of the pI standards are shown at left.

(B) PCR analysis of SLA in different B. oleracea lines. Genomic DNA was extracted and PCR amplification was carried out with three oligonucleotides corresponding to $S L A_{S c}$ sequences (OSLA1, OSLA2, and OSLA3; see Figure 1). The PCR products were separated on an agarose gel and stained with ethidium bromide. A 798-bp PCR product is expected if the $S L A$ gene contains an insert in the same position as that in $S L A_{S c}$ (amplification with oligonucleotides OSLA1 and OSLA2), whereas a 612-bp product is expected if an SLA gene with no insert is present (amplification with oligonucleotides OSLA1 and OSLA3). DNA was from B. oleracea var acephala lines homozygous for the $S_{5}, S_{2}$, and $S_{S c}$ (the P57Sc line) haplotypes and from the B. oleracea var botrytis lines 41865, 24062, 11035, 17144, 40443, 14518, and 22495. Numbers at left indicate the lengths of PCR products in base pairs.

Figure 5. RNA gel blot analysis of SLA transcription.

Each lane corresponds to $16 \mu \mathrm{g}$ of poly $(\mathrm{A})^{+}$RNA from anthers of a line homozygous for the $S_{2}$ haplotype $\left(S_{2}\right)$, the P57Sc line $\left(S_{S c}\right)$, or B. oleracea var botrytis line 11035 (11035) at either the unicellular/bicellular stages of microspore development (1) or at the tricellular stage (2). 
(A) The RNA gel blot was probed with a 1260-bp EcoRV-BamHI fragment from $S L A_{S c}$ (SLA probe in Figure 1), and the autoradiograph was exposed for 10 days. (B) The same RNA gel blot probed with a Brassica actin cDNA probe. The autoradiograph was exposed for $6 \mathrm{~h}$. Numbers at left indicate the lengths of RNA molecular markers in kilobases (kb).

Figure 6. DNA blot analysis of the SLA gene.

DNA gel blot of HindIII restriction endonuclease digested genomic DNA from B. oleracea var acephala lines homozygous for the $S_{3}, S_{2}$ and $S_{S c}$ (the P57Sc line) haplotypes and from the B. oleracea var botrytis lines $41865,24062,11035,17144,40443,14518$, and 22495 . A 1260-bp EcoRV-BamHI fragment from $S L A_{S c}$ (SLA probe in Figure 1) was used as a probe. Numbers at left indicate the lengths of hybridizing fragments in kilobases. 


\section{TABLES}

Table 1. Origins and SI phenotypes of the B. oleracea var botrytis lines used in this study

\begin{tabular}{|c|c|c|c|c|c|c|}
\hline \multirow{2}{*}{ Line } & \multirow{2}{*}{ Type } & \multirow{2}{*}{$\begin{array}{c}\text { Number of } \\
\text { generations of } \\
\text { self-pollination }\end{array}$} & \multicolumn{3}{|c|}{ Pollen tube mean score ${ }^{\mathrm{a}}$} & \multirow{2}{*}{ Phenotype $^{b}$} \\
\hline & & & mean & $\min$. & $\max$. & \\
\hline 11035 & Autumn & 5 & 0.1 & 0.0 & 0.3 & SI \\
\hline 24062 & Autumn & 14 & 0.3 & 0.3 & 0.3 & SI \\
\hline 41865 & Autumn & 9 & 0.1 & 0.0 & 0.3 & SI \\
\hline 17144 & Autumn & 6 & 0.9 & 0.0 & 1.7 & SI \\
\hline 40443 & Autumn & 7 & 0.7 & 0.0 & 1.7 & SI \\
\hline 14518 & Winter & 4 & 4.9 & 4.7 & 5.0 & $\mathrm{SC}$ \\
\hline 22495 & Winter & 9 & 5.4 & 4.0 & 6.8 & $\mathrm{SC}$ \\
\hline
\end{tabular}

${ }^{\text {a }}$ Pollen tube mean scores were determined by aniline blue staining of pistils 24 hours after pollination. Three pistils were scored at one time, and between two to five triplets of pistils were scored for each plant. Nine scores were defined: 0,0 tubes; 1,1 or 2 tubes; 2, 3-5 tubes; 3, 6-9 tubes; 4, 10-14 tubes; 5, 15-25 tubes; 6, 26-50 tubes; 7, 51-100 tubes; $8=>100$ tubes. The mean score was determined from all the pistils tested for a particular plant, min. and max. scores refer to minimum and maximum scores, respectively, obtained from a triplet of pistils for each plant.

b The SI phenotype based on pollen tube mean scores: SI, self-incompatible; SC, selfcompatible. 
Table 2. PCR detection of $S L A$ in progeny issued from self-fertilization of the seven $B$. oleracea var botrytis plants used in this study

\begin{tabular}{ccccc}
\hline Line $^{\mathrm{a}}$ & Phenotype $^{\mathrm{b}}$ & $\begin{array}{c}\text { Number of } \\
\text { progeny } \\
\text { tested }\end{array}$ & $\begin{array}{c}\text { Number of } \\
\text { PCR positive } \\
\text { progeny }^{\mathrm{c}}\end{array}$ & $\begin{array}{c}\text { Probability } \\
\text { that parent was } \\
\text { homozygous }\end{array}$ \\
\hline 41865 & SI & 29 & 29 & $99.97 \%$ \\
24062 & SI & 17 & 17 & $99.2 \%$ \\
11035 & SI & 17 & 17 & $99.2 \%$ \\
17144 & SI & 10 & 10 & $94.4 \%$ \\
40443 & SI & 15 & 15 & $98.7 \%$ \\
14518 & SC & 13 & 13 & $97.6 \%$ \\
22495 & SC & 16 & 16 & $99.0 \%$ \\
\hline
\end{tabular}

${ }^{\text {a }}$ Genomic DNA was extracted from each of the plants tested and a fragment of SLA was amplified using oligonucleotides OSLA1, OSLA2, and OSLA3 (see Figure 1).

${ }^{\mathrm{b}}$ The SI phenotype based on the pollen tube mean scores (see Table 1): SI, self-incompatible; SC, self-compatible.

${ }^{c}$ A 798-bp product was amplified from genomic DNA of these plants indicating the presence of an SLA gene interrupted by an insertion. 